\title{
In-situ XRD studies of nanocrystallization of Fe-based metallic glass: Comparative study by reciprocal and direct space methods
}

\author{
Jozef Bednarčík, ${ }^{a *} \check{S}_{\text {tefan Michalik, }}^{b}$ Vladimír Kolesár, ${ }^{c}$ Uta Rütt, ${ }^{a}$ and Hermann Franz ${ }^{a}$
}

DOI: 10.1039/C3CP44445G

Structural changes occurring in an $\mathrm{Fe}_{72.5} \mathrm{Cu}_{1} \mathrm{Nb}_{2} \mathrm{Mo}_{2} \mathrm{Si}_{15.5} \mathrm{~B}_{7}$ alloy during a combination of constant rate heating (20 K/min) and isothermal holding at 500 and $520{ }^{\circ} \mathrm{C}$ were investigated using in-situ high-energy X-ray diffraction. We found that the ferromagnetic-to-paramagnetic transition of an amorphous phase is revealed as a change of the slope of the thermal expansion curve when heating a sample with a constant rate up to $520^{\circ} \mathrm{C}$. Real space analysis by means of atomic pair distribution function (PDF) demonstrated that the rate and extent of the thermal expansion strongly depends on the interatomic separation. PDF proved to be a reliable method for the description of crystallization kinetics. Further it allows determination of sizes of ultrafine nanocrystals with grain sizes well below $8 \mathrm{~nm}$ and thus makes observation of early stages of nanocrystallization possible. Following grain growth kinetics during isothermal annealing at 500 and $520^{\circ} \mathrm{C}$ we found that the activation energy of the process is $357 \pm 12 \mathrm{~kJ} / \mathrm{mol}$.

\section{Introduction}

Nanocrystalline Fe-based alloys exhibit outstanding soft magnetic properties characterized by a very low coercivity and a very high saturation magnetization ${ }^{1}$. Soft magnetic nanostructured materials can be obtained from many conventional metallic glasses (usually prepared by melt spinning method) by a controlled crystallization at temperatures slightly below primary crystallization ${ }^{2}$. Optimized alloy compositions like $\mathrm{Fe}_{73} \mathrm{Cu}_{1} \mathrm{Nb}_{3} \mathrm{Si}_{16} \mathrm{~B}_{7}$ moreover reveal near-zero saturation magnetostriction and meanwhile have successfully entered into application under the trademarks FINEMET $^{3}$ or VITROPERM $800^{4}$. These materials exhibit a microstructure in which ultrafine grains of BCC FeSi (typical grain size 10-15 nm) are randomly distributed within a residual amorphous matrix. The ferromagnetic character of the amorphous matrix enables exchange coupling between ferromagnetic nanocrystals, which yield a severe reduction of the average magnetocrystalline anisotropy ${ }^{5,6}$. Since magnetic properties of Febased nanocrystalline materials are strongly related to their microstructure (mostly grain size distribution) many authors studied kinetics of their crystallization ${ }^{2,7,8}$. Recently, significant advances have been made using the atomic pair distribu-

${ }^{a}$ Deutsches Elektronen-Synchrotron (DESY), Notkestr. 85, 22607 Hamburg, Germany. Fax: +49 (0)40 8994 2720; Tel: +49 (0)40 8998 1861; E-mail: Jozef.Bednarcik@desy.de

${ }^{b}$ P.J. Safarik University in Košice, Institute of Physics, Park Angelinum 9, 04154 Košice, Slovakia. Currently affiliated with: Institute of Physics of the ASCR, v. v. i., Na Slovance 2, 18221 Praha 8, The Czech Republic.

${ }^{c}$ Slovak University of Technology, Institute of Materials Science, Bottova 25, 91724 Trnava, Slovakia. tion function (PDF) analysis of powder diffraction data coupled with the use of advanced X-ray and neutron sources and fast computers $^{9-12}$.

The aim of the present work is the investigation of structural changes occurring in $\mathrm{Fe}_{72.5} \mathrm{Cu}_{1} \mathrm{Nb}_{2} \mathrm{Mo}_{2} \mathrm{Si}_{15.5} \mathrm{~B}_{7}$ alloy during combination of constant heating rate $(20 \mathrm{~K} / \mathrm{min})$ and an isothermal holding at 500 and $520^{\circ} \mathrm{C}$ by means of in-situ high-energy X-ray diffraction. Evaluation of X-ray diffraction data is done by two approaches, in reciprocal space by analysis of diffraction profiles and in real space by pair distribution function. One of the main goals is to demonstrate the benefits of using in-situ PDF techniques for the investigation of crystallization kinetics of nanocrystalline materials.

\section{Experiment}

The amorphous alloy with nominal composition $\mathrm{Fe}_{72.5} \mathrm{Cu}_{1} \mathrm{Nb}_{2} \mathrm{Mo}_{2} \mathrm{Si}_{15.5} \mathrm{~B}_{7}$ (at. \%) was prepared in the form of thin ribbons using single-roller melt-spinning technique. The width and thickness of the ribbons were $9 \mathrm{~mm}$ and $30 \mu \mathrm{m}$, respectively. A differential scanning calorimeter (NETZSCH DSC 404C) was used to detect the crystallization behavior of as-prepared ribbons under a continuous argon flow at a heating rate of $20 \mathrm{~K} / \mathrm{min}$. The thermomagnetic curve was traced at a heating rate of $10 \mathrm{~K} / \mathrm{min}$ using a Faraday magnetic balance. In-situ annealing experiments using a high-energy photon beam $(80.09 \mathrm{keV})$ were performed at the undulator beamline P07 (Physics Hutch) of the electron storage ring PETRA III (Hamburg, Germany). Few pieces of ribbon were stuck together and put inside a quartz capillary 
(wall thickness $20 \mu \mathrm{m}$ ) with a diameter of $1.2 \mathrm{~mm}$ and placed inside a Linkam THMS600 hot stage. The samples were heated in an argon atmosphere. Each in-situ experiment started with i) heating at a constant rate of $20 \mathrm{~K} / \mathrm{min}$ up to the selected temperature and followed by ii) isothermal annealing at that temperature for at least $100 \mathrm{~min}$. Two different annealing temperatures were chosen, namely $500^{\circ} \mathrm{C}$ and $520^{\circ} \mathrm{C}$ (we refer to as Vit500 and Vit520). X-ray diffraction patterns were continuously recorded in transmission mode using a two-dimensional detector (Perkin Elmer 1621, 2048×2048 pixels, pixel size $200 \times 200 \mu \mathrm{m}^{2}$ ). The temporal resolution between two consecutive diffraction patterns was 12 seconds. The wavelength and beam size of monochromatic photon beam were set to $0.1548 \AA(80.09 \mathrm{keV})$ and $0.5 \times 0.5 \mathrm{~mm}^{2}$, respectively. The sample-to-detector distance and the orthogonality of the detector with respect to the incident photon beam were calibrated using the reference material $\mathrm{LaB}_{6}$ (NIST 660a). Two-dimensional X-ray diffraction patterns were radially integrated using the programme FIT2D ${ }^{13}$.

\section{Background on data evaluation}

In case of an angle-dispersive X-ray diffraction experiment the incident monochromatic photon beam with wavelength $\lambda$ is diffracted under an angle $2 \theta_{h k l}$ by the set of lattice planes having an interplanar spacing $d_{h k l}$. This is well known as Bragg's law

$$
2 d_{h k l} \sin \left(\theta_{h k l}\right)=\lambda .
$$

Bragg's law, as stated above, can be used to obtain the lattice parameter of for example a particular cubic system through the following relation

$$
d_{h k l}=\frac{a}{\sqrt{h^{2}+k^{2}+l^{2}}}
$$

where $a$ is the lattice parameter of the cubic crystal and $h, k$ and $l$ are the Miller indices of the Bragg plane. The simple relationship between diffraction angle $\theta$, reciprocal $q$ and real $d$-space is then

$$
q=\frac{2 \pi}{d}=\frac{4 \pi \sin (\theta)}{\lambda} .
$$

The angular distribution of scattered intensities around a Bragg peak can be satisfactorily described by the pseudoVoigt function $I_{p V}(2 \theta)$ having the analytical form

$$
I_{p V}(2 \theta)=A[\eta G(2 \theta)+(1-\eta) L(2 \theta)],
$$

where $A$ is the amplitude, $G(2 \theta)=\exp \left[-(\ln 2) k^{2}\right]$ represents the Gaussian and $L(2 \theta)=\left(1+k^{2}\right)^{-1}$ the Lorentzian part, with $k=2\left(2 \theta-2 \theta_{h k l}\right) / \beta$, in which $\beta$ is the full-width at halfmaximum (FWHM), $\eta$ the Gaussian content and $2 \theta_{h k l}$ the position of the Bragg peak. The mixing factor $\eta$ may have any value in the interval $\langle 0,1\rangle$.

\subsection{Diffraction profile analysis}

Diffraction profile parameters such as Bragg peak intensity, position and width carry important information about the sample's microstructure. The peak position $\theta_{h k l}$ is closely related with the lattice parameter $a$. Combining equations (1) and (2) and plotting $\lambda /\left(2 \sin \theta_{h k l}\right)$ as a function of $1 / \sqrt{h^{2}+k^{2}+l^{2}}$ one gets from the slope of such dependence the value of lattice parameter $a$. The width of the Bragg reflection $\beta_{h k l}$ contains useful information about the mean size of coherently scattering domains. In 1918 Scherrer $^{14}$ derived an equation which relates the mean (volume average) crystallite size $D$ of a powder to the broadening $\beta_{h k l}^{D}$ of its powder diffraction peaks

$$
\beta_{h k l}^{D}=\frac{K \lambda}{D \cos \theta_{h k l}}
$$

where $K$ is the Scherrer constant (in our calculation of grain sizes, $K$ was equal to unity). It should be noted that the Scherrer equation as stated above ignores effects of strain on diffraction line broadening.

The Williams-Hall method ${ }^{15}$ relies on the principle that the approximate formulae for size broadening $\beta_{h k l}^{D}$ and strain broadening $\beta_{h k l}^{\varepsilon}$ show different dependence on Bragg angle $\theta_{h k l}$ and thus the total line broadening $\beta_{h k l}$ can be expressed in the form

$$
\beta_{h k l}=\beta_{h k l}^{\varepsilon}+\beta_{h k l}^{D}=C \varepsilon \tan \theta_{h k l}+\frac{K \lambda}{D \cos \theta_{h k l}}
$$

where $\varepsilon$ is the mean strain and $C$ represents the constant which depends on the assumptions made concerning the nature of the inhomogeneous strain, but is typically equal to $4^{16}$.

In order to get the correct information about the mean grain size determined from the peak broadening, it is necessary to correct the measured line widths $\beta_{\text {exp }}$ for the instrumental contribution $\beta_{\text {ins. }}$. The so called Instrumental Resolution Function (IRF) is usually determined by measuring standard materials such as $\mathrm{LaB}_{6}$ at the same experimental and geometrical conditions as the studied material. The IRF expresses the evolution of the line broadening as a function of the diffraction angle $2 \theta$ and is usually fitted to the Caglioti formula ${ }^{17}$

$$
\beta_{\text {ins }}^{2}=U \tan ^{2} \theta+V \tan \theta+W
$$

where $U, V$ and $W$ are fitting parameters characterizing the instrument. When describing diffraction profiles by a Lorentzian profile function (putting $\eta=0$ in equation (4)) correction of the line widths is performed in the following way $\beta=\beta_{\text {exp }}-\beta_{\text {ins }}$, whereas using Gaussian profiles (putting $\eta=1$ in equation (4)) yields the correction $\beta^{2}=\beta_{\text {exp }}^{2}-\beta_{\text {ins }}^{2}$. It should be noted here that the Williams-Hall plot defined by equation (6) is appropriate for situations when pure Lorentzian profiles are considered. If pure Gaussians profiles are considered then all terms in equation (4) should be squared ${ }^{16}$. 
The integral intensity of the Bragg peak is proportional to the amount of the corresponding crystalline phase and can be used to evaluate its relative fraction within a multi-phase alloy system. According to work of Riello et al. ${ }^{18}$ the amount of crystalline phase $X_{\text {cryst }}$ can be calculated as

$$
X_{\text {cryst }}=\frac{M_{\text {cryst }}}{M_{\text {total }}} \frac{\sum_{i=1}^{n_{\text {cryst }}} m_{i}}{\sum_{i=1}^{n_{\text {sample }}} m_{i}}
$$

where $M_{\text {cryst }}$ and $M_{\text {total }}$ are defined in the following way

$$
\begin{gathered}
M_{\text {cryst }}=\frac{\int_{q_{\min }}^{q_{\max }} I_{\text {cryst }}(q) q^{2} d q}{\sum_{i=1}^{n_{\text {cryst }}} \int_{q_{\min }}^{q_{\max }}\left[\left|f_{i}(q)\right|^{2}+I_{i}^{\text {inc }}(q)\right] q^{2} d q} \\
M_{\text {total }}=\frac{\int_{q_{\min }}^{q_{\max }} I_{\text {total }}(q) q^{2} d q}{\sum_{i=1}^{n_{\text {sample }}} \int_{q_{\min }}^{q_{\max }}\left[\left|f_{i}(q)\right|^{2}+I_{i}^{\text {inc }}(q)\right] q^{2} d q}
\end{gathered}
$$

in which $I_{\text {cryst }}$ is the intensity contribution of the crystalline phase to the global intensity $I_{\text {total }}$ of the XRD pattern, $I_{i}^{i n c}(q)$ is the incoherent scattering of $i$-th atom calculated using the Balyuzi formula ${ }^{19}, f_{i}(q)$ is the tabulated atomic scattering factor ${ }^{20}$ of the $i$-th atom, $n_{\text {cryst }}$ and $n_{\text {sample }}$ represent the number of atoms in the unit cell of the crystalline phase and the number of atoms in the composition unit of the sample, respectively and $m_{i}$ is the atomic weight of the $i$-th atom.

\subsection{Structure factor and pair distribution function}

The total structure factor, $S(q)$, was obtained using standard procedures described in ${ }^{21,22}$. Considering background scattering, $b g(q)$, and sample absorption, $A$, the observed intensity $I^{o b s}(q)$ was corrected in the following way $I^{c o r}(q)=$ $\left[I^{o b s}(q)-b g(q)\right] / A$. The absorption of the sample at a given energy was determined by measuring the incoming and transmitted beam intensity using a beam monitor. To suppress fluorescence background we put a $1 \mathrm{~mm}$ thick aluminium sheet on the front face of the 2D detector. The corrected intensity $I^{c o r}(q)$ can be expressed by the relation

$$
\alpha I^{c o r}(q)=I_{e u}^{c o h}(q)+I_{e u}^{i n c}(q)+I_{e u}^{m u l}(q)
$$

where $I_{e u}^{c o h}(q), I_{e u}^{i n c}(q)$ and $I_{e u}^{m u l}(q)$ are the coherent, incoherent (Compton) ${ }^{23}$ and multiple scattering intensities, respectively, in electron units, and $\alpha$ is a conversion factor which is often called the normalization constant. To place data on an absolute scale, we employed the high-angle region method ${ }^{22}$ in which the normalization constant is calculated as

$$
\alpha=\frac{\int_{q_{\operatorname{mid}}}^{q_{\max }}\left[\left\langle f^{2}\right\rangle+I_{e u}^{i n c}(q)+I_{e u}^{\operatorname{mul}}(q)\right] d q}{\int_{q_{\operatorname{mid}}}^{q_{\max }}\left[I^{\text {cor }}(q)\right] d q}
$$

where $q_{\text {max }}$ and $q_{\text {mid }}$ are the upper limit and an appropriate high value in the measured range of $q$ respectively. Generally, the adopted value of $q_{m i d}$ is $8-10 \AA^{-1}$. Further we assumed isotropic fluorescence intensity which was adjusted to a constant value based on the behavior of the resulting structure factor $S(q)$. Once selected, the same fluorescence intensity was used for all data sets.

The total structure factor $S(q)$ was obtained from the normalized elastically scattered intensity, $I_{e u}^{c o h}(q)$ using the FaberZiman formalism ${ }^{24}$

$$
S(q)=1+\frac{I_{e u}^{c o h}(q)-\left\langle f^{2}\right\rangle}{\langle f\rangle^{2}}
$$

where $\left\langle f^{2}\right\rangle=\sum_{i=1}^{N} c_{i} f_{i}^{2}(q),\langle f\rangle^{2}=\left[\sum_{i=1}^{N} c_{i} f_{i}(q)\right]^{2}$ in which $c_{i}$ are the atomic concentration of the atomic species of type $i(i=$ $\mathrm{Fe}, \mathrm{Si}, \mathrm{Cu}, \mathrm{Mo}, \mathrm{Nb}, \mathrm{B})$. The corresponding reduced pair distribution function, $G(r)$, can be obtained through a sine Fourier transformation

$$
G(r)=4 \pi r\left[\rho(r)-\rho_{0}\right]=\frac{2}{\pi} \int_{0}^{\infty} q[S(q)-1] \sin (r q) d q
$$

where $\rho(r)$ and $\rho_{0}$ are the local and average atomic number densities, respectively, and $r$ is the radial distance. Since $\rho(r)$ becomes zero below a certain $r_{\text {min }}$, the average atomic number density $\rho_{0}$ can be easily obtained by linear fit of $G(r)$ 's low $r$ part (where $r \leq r_{\text {min }}$ ) to $-4 \pi \rho_{0} r$. From $G(r)$ the radial distribution function, $R D F(r)$, can be calculated by

$$
R D F(r)=4 \pi r^{2} \rho(r)=4 \pi r^{2} \rho_{0}+r G(r) .
$$

The average coordination number, $N$, around any given atom in a spherical shell between radii $r_{1}$ and $r_{2}$ can be calculated as

$$
N=\int_{r_{1}}^{r_{2}} R D F(r) d r=\frac{4 \pi \rho_{0}}{3}\left(r_{2}^{3}-r_{1}^{3}\right)+\int_{r_{1}}^{r_{2}} r G(r) d r .
$$

\section{Results and discussion}

The room temperature X-ray diffraction measurements confirmed the fully amorphous character of the $\mathrm{Fe}_{72.5} \mathrm{Cu}_{1} \mathrm{Nb}_{2} \mathrm{Mo}_{2} \mathrm{Si}_{15.5} \mathrm{~B}_{7}$ ribbons in the as-quenched state. $\mathrm{X}$-ray diffraction patterns can be characterized by the first sharp diffraction peak (FSDP) located at $Q_{1}=3.08 \AA^{-1}$ $(2 \theta=4.33 \mathrm{deg}$.$) and relatively fast decaying oscillations$ visible up to $17 \AA^{-1}$. Differential calorimetry measurements showed that the investigated alloy crystallizes in two steps. The primary and secondary crystallization occur at $T_{x 1}=516^{\circ} \mathrm{C}$ and $T_{x 2}=688^{\circ} \mathrm{C}$, respectively. The initial decrease of the magnetization with increasing temperature (see Fig.1) is related to the transition of the amorphous phase from the ferromagnetic to the paramagnetic state. The first derivative yields the Curie temperature of the amorphous phase $T_{C}=325^{\circ} \mathrm{C}$. 


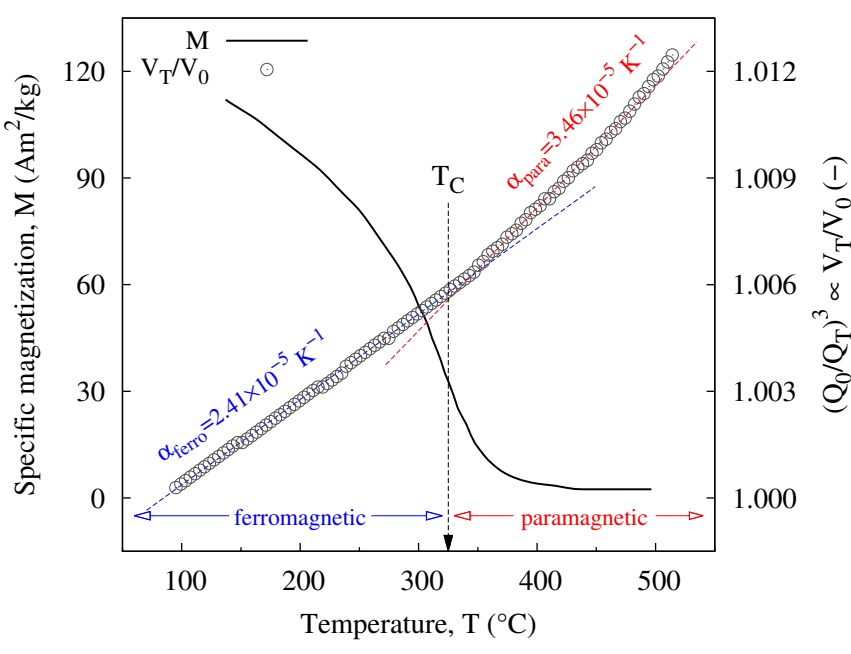

Fig. 1 (Color online) Temperature behavior of volume changes as derived from the relative change of the FSDP during constant rate heating at $20 \mathrm{~K} / \mathrm{min}$ up to $520^{\circ} \mathrm{C}$. Blue and red dashed lines emphasize linear trends of thermal expansion curve. The solid black line shows the temperature dependence of the magnetization.

\subsection{Reciprocal space analysis}

4.1.1 Thermal expansion behavior In the first part we focus on structural changes occurring in the amorphous alloy $\mathrm{Fe}_{72.5} \mathrm{Cu}_{1} \mathrm{Nb}_{2} \mathrm{Mo}_{2} \mathrm{Si}_{15.5} \mathrm{~B}_{7}$ during constant rate heating. To obtain quantitative results about the thermal expansion of the amorphous phase from X-ray diffraction data the profile of the FSDP was modelled assuming a linear background and a pseudo-Voigt function described by equation (4). As proposed by Yavari and co-workers ${ }^{25}$ the third power of the principal peak position $Q_{1}$ scales with the coefficient of the volume thermal expansion $\alpha_{t h}$ of an isotropic amorphous solid as

$$
\left\{\frac{Q_{1}\left(T_{0}\right)}{Q_{1}(T)}\right\}^{3}=\frac{V(T)}{V\left(T_{0}\right)}=1+\alpha_{t h}\left(T-T_{0}\right),
$$

where $V(T) / V\left(T_{0}\right)$ is the reduced mean atomic volume at temperature $T$, with the reference temperature $T_{0}$ (in our study $100^{\circ} \mathrm{C}$ ). It should be noted here that for isotropic materials, the linear thermal expansion coefficient $\gamma_{t h}$ is one third of the volumetric coefficient $\alpha_{t h}$, i.e. $\alpha_{t h}=3 \gamma_{t h}$. Figure 1 shows the thermal expansion curve as determined from the relative change of the FSDP. It is interesting to note that the thermal expansion curve shows a distinct change of the slope at the Curie point $T_{C}$. The coefficient of volume thermal expansion in the ferromagnetic state is $\alpha_{\text {ferro }}=(2.41 \pm 0.05) \times$ $10^{-5} \mathrm{~K}^{-1}$, whereas the paramagnetic state reveals a significantly larger value $\alpha_{\text {para }}=(3.46 \pm 0.05) \times 10^{-5} \mathrm{~K}^{-1}$. This means that the magnetic interaction forces present below $T_{C}$ compete with forces associated with thermal vibrations and effectively reduce the expansion of interatomic distances. On

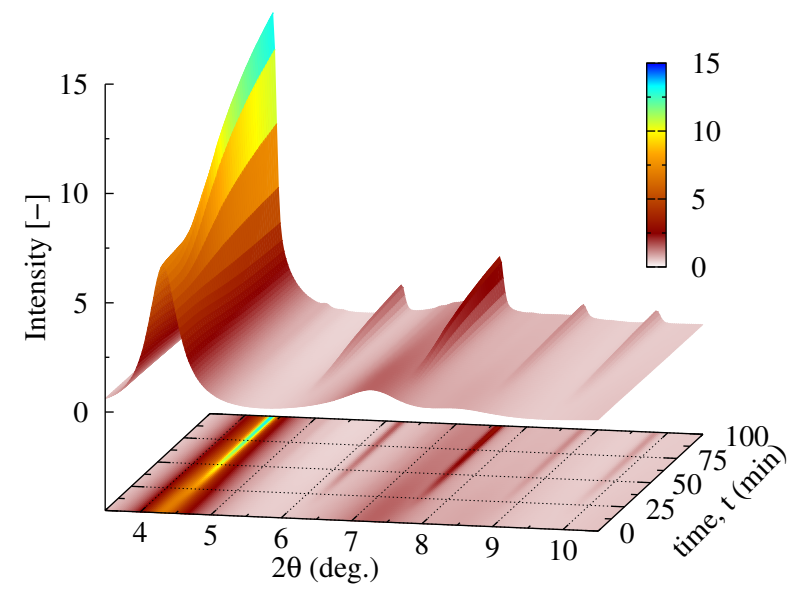

Fig. 2 (Color online) Series of XRD patterns collected as a function of time $t$ during isothermal annealing at $500{ }^{\circ} \mathrm{C}$.

the other hand, at the moment when the temperature reaches $T_{C}$, ferromagnetic interactions are switched off and atoms can behave more freely. Michalik and co-workers ${ }^{26}$ have recently shown that the change of the slope observed on thermal expansion curves of $\mathrm{Fe}_{(73.5-x)} \mathrm{Mn}_{x} \mathrm{Si}_{13.5} \mathrm{Cu}_{1} \mathrm{Nb}_{3} \mathrm{~B}_{9}(\mathrm{x}=1,3,5,7$ and 9) metallic glasses is closely related to the ferromagneticto-paramagnetic transition of the amorphous phase.

4.1.2 Crystallization kinetics In the next part, the kinetics of crystallization at two different temperatures, namely at 500 and $520^{\circ} \mathrm{C}$, is described in detail. Figure 2 shows series of XRD patterns recorded as a function of time during in-situ isothermal annealing at $500{ }^{\circ} \mathrm{C}$. One can readily see that after reaching the temperature of $500{ }^{\circ} \mathrm{C}$ the sample gradually devitrifies and Bragg reflections belonging to $\mathrm{Fe}_{3} \mathrm{Si}$ become clearly visible. The times required for the initiation of crystallization are $12.5 \mathrm{~min}$ and $2.2 \mathrm{~min}$ for the Vit500 and Vit520 sample, respectively. The cubic $\mathrm{Fe}_{3} \mathrm{Si}$ phase is the only phase formed during devitrification and it is typical for $\mathrm{Fe}-\mathrm{Si}-\mathrm{Cu}-\mathrm{Nb}-\mathrm{B}$ based amorphous alloys ${ }^{2}$. Detailed information about the temporal evolution of the crystallized fraction, mean grain size and lattice parameter of the cubic $\mathrm{Fe}_{3} \mathrm{Si}$ phase during isothermal annealing were evaluated from a series of XRD patterns. The crucial point in performing a quantitative analysis was to reasonably separate the amorphous and the crystalline contribution from the total scattering. The profile fitting was realized in the following way. The contribution from the crystalline $\mathrm{Fe}_{3} \mathrm{Si}$ phase was expressed as a sum of seven Gaussian functions, describing the (200), (400), (422), (440), (620), (444) and (642) Bragg reflections. The XRD patterns of Vit500 and Vit520 samples detected after $11.6 \mathrm{~min}$ and $0.0 \mathrm{~min}$ (just before crystallization occurred), respectively, were multiplied with a scaling parameter and used for modelling of the amorphous contribution in the respective run. In 


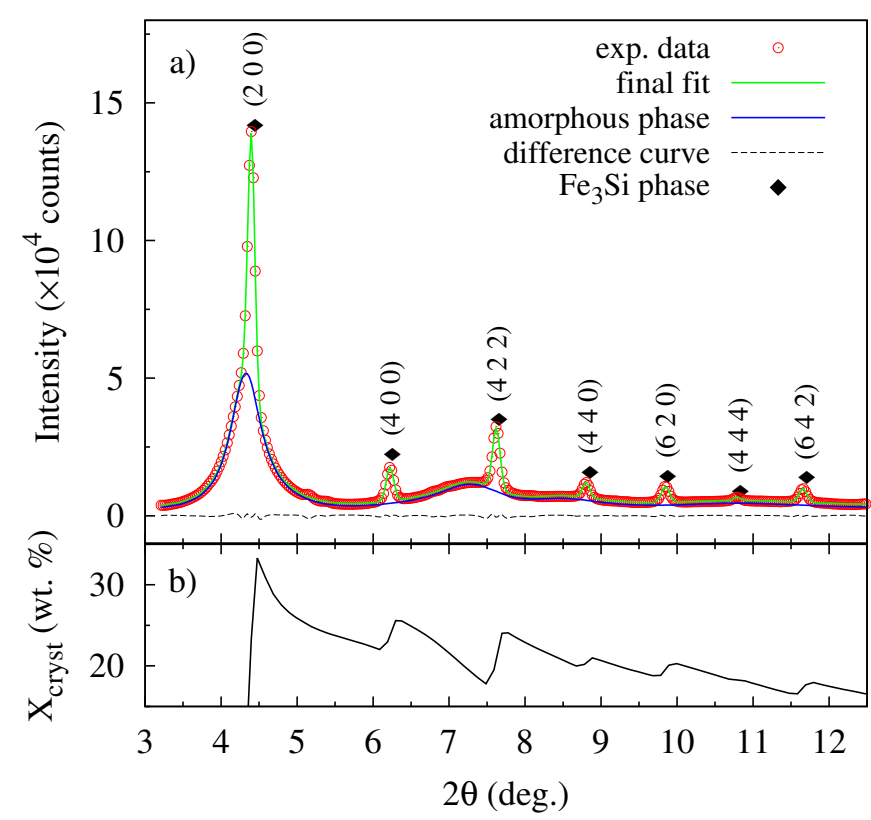

Fig. 3 (Color online) a) Comparison between the best-fit model and the experimental XRD pattern of the sample annealed at $500{ }^{\circ} \mathrm{C}$ for $100 \mathrm{~min} . \mathrm{b})$ The amount of crystalline phase calculated as a function of the upper limit of the $2 \theta$ range (the lower limit was fixed to $3 \mathrm{deg}$ ) using equation (8).

total 25 parameters were needed to refine the XRD profile in a $2 \theta$ interval ranging from 3 up to 12.5 degrees. Three profile parameters were needed for every Bragg peak (amplitude $A$, peak position $\theta_{h k l}$ and full-width at half-maximum $\beta_{h k l}$ ). Another three parameters were used to account for a quadratic polynomial describing background and one scaling parameter was used to adjust the contribution from the amorphous matrix. A similar approach was previously used by Michalik et $a l .{ }^{27}$ when studying the crystallization behavior of glassy microwires using in-situ XRD. As can be seen from Fig.3a, the proposed model satisfactorily describes the XRD profile.

The fitted profiles of crystalline and amorphous components, which contribute to the total scattering, were weighted following the work of Riello et al. ${ }^{18}$. The amount of crystallized fraction was calculated based on refined XRD profiles using equation (8). Figure 3 b shows the crystalline amount as a function of the upper limit of $2 \theta$ used for its evaluation (the lower limit of $2 \theta$ was fixed at 3 degrees). It is seen that the relative fraction of the crystalline component calculated from the XRD profile strongly depends on the $2 \theta$ interval used. When studying devitrification of metallic glasses authors of many works tend to use a simple approach and refine only narrow part of the XRD profile, mostly in the vicinity of the first sharp diffraction peak ${ }^{28,29}$. Then the intensity profile is fitted with two peak functions, one representing an amorphous
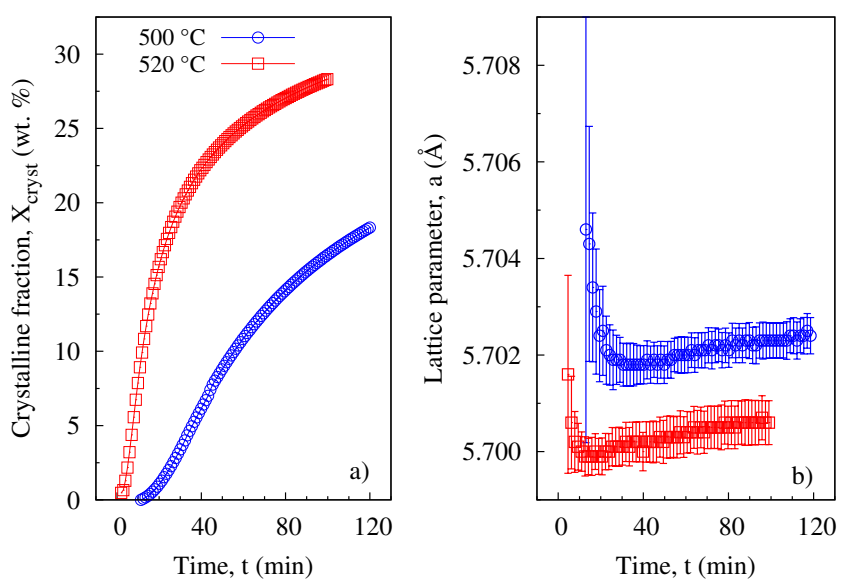

Fig. 4 (Color online) Temporal evolution of a) crystalline fraction $X_{\text {cryst }}$ and b) lattice parameter $a$ of the $\mathrm{Fe}_{3} \mathrm{Si}$ phase during isothermal annealing at 500 and $520^{\circ} \mathrm{C}$.

component and a second one corresponding to a Bragg peak. Lorentz, Gaussian or Pseudo-Voigt function are usually used to describe intensity profiles. On the other hand some authors refine the XRD profiles on $2 \theta$ interval as large as possible ${ }^{18,30}$. Actually this approach is followed in this work. Figure $4 \mathrm{a}$ shows the temporal evolution of the crystalline weight fraction $X_{\text {cryst }}$ during isothermal annealing at 500 and $520^{\circ} \mathrm{C}$. In case of the Vit500 sample it takes about $12.5 \mathrm{~min}$ until tiny traces of $\mathrm{Fe}_{3} \mathrm{Si}$ phase appear in the diffraction patterns. The sample Vit520 is crystallizing almost instantly with incubation time of only $2.2 \mathrm{~min}$. Higher temperature of isothermal annealing implies faster crystallization kinetics. Nevertheless the crystalline fraction does not saturate even after $100 \mathrm{~min}$ of annealing at $520^{\circ} \mathrm{C}$. The time dependence of the lattice parameter $a$ for samples Vit500 and Vit520 is shown in Figure 4b.

Figure 5 shows the time evolution of the mean grain size during isothermal annealing at 500 and $520^{\circ} \mathrm{C}$. The mean grain size was calculated either by the Scherrer equation ${ }^{14}$ (Fig.5a) or by the Williamson-Hall method ${ }^{15}$ (Fig.5b). In the case of the Scherrer approach, the mean grain size was calculated for every indexed Bragg peak shown in Fig.3 separately. Then the average value was calculated and is plotted in Fig.5. The error was estimated as standard deviation. It should be noted here that the Bragg reflection (444) was omitted from the calculations since it was small and imposed an error on the final values. Qualitatively speaking, both approaches, the Scherrer formula and the Williamson-Hall plot, brought very similar results. The main disparity between both methods is in the absolute values of the obtained sizes. It seems that values of the mean grain size obtained by the Williamson-Hall method are systematically larger by $20 \AA$ than the ones calculated by the Scherrer formula. This is due to the fact that the Scherrer equation does not account for line broadening due to 

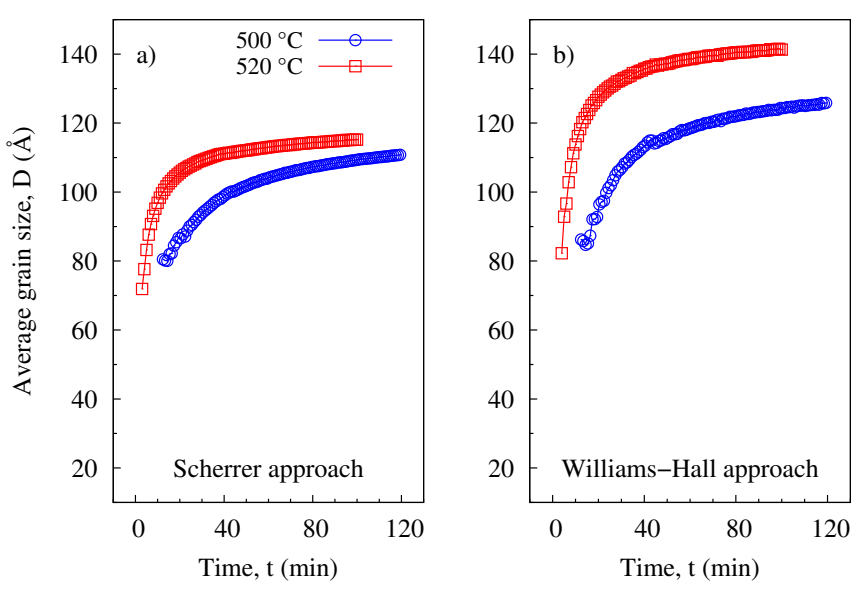

Fig. 5 (Color online) Comparison of the mean grain size $D$ calculated by a) Scherrer equation and b) Williams-Hall method. The uncertainty in determination of the mean grain size is $\pm 7 \AA$.

strain and therefore systematically underestimates grain size values. As can be seen from Fig.5 the size of crystallites increases with the annealing time and higher temperature implies faster grain growth. Apparently, the growth of crystallites is much more pronounced in the first minutes of annealing. Longer annealing times mean gradual decrease of grain growth dynamics.

\subsection{Real space analysis}

Figure 6 shows the structure factor $S(q)$ and the corresponding reduced pair distribution function $G(r)$ for the $\mathrm{Fe}_{72.5} \mathrm{Cu}_{1} \mathrm{Nb}_{2} \mathrm{Mo}_{2} \mathrm{Si}_{15.5} \mathrm{~B}_{7}$ alloy in the as-quenched state. Fitting $-4 \pi \rho_{0} r$ to the $G(r)$ for $r<=1.9 \AA^{-1}$ we determined the value of the mean atomic density $\rho_{0}=0.082 \AA^{-3}$. The average coordination number $N$ in the first coordination shell (between radii 2.1 and $3.5 \AA$ ) was calculated according equation (16) and is 13.5. Due to the highest concentration and $\mathrm{X}$-ray scattering power of $\mathrm{Fe}$ atoms, $\mathrm{Fe}-\mathrm{Fe}$ atomic pairs are the ones which determine the shape of the first coordination shell appearing in $G(r)$. The mean atomic distance between two neighboring $\mathrm{Fe}$ atoms is $r_{\mathrm{Fe}-\mathrm{Fe}}=2.64 \AA$.

4.2.1 Thermal expansion behavior In the previous section 4.1.1 we presented thermal expansion behavior data evaluated by the relative shift of the FSDP in reciprocal space. In order to see what are corresponding distances in real space, we performed a Fourier transformation of the FSDP. As can be seen from Fig.6, the Fourier transform of the FSPD is perfectly matching oscillations on $G(r)$ for distances $r$ larger than $8 \AA$. This shows that the FSDP in reciprocal space is closely related to the medium range order (in the range 8-20 $\mathrm{A}$ ). Similar findings were recently reported in La-based metallic glass ${ }^{12}$. Further we evaluated the relative shift of coordination shells as

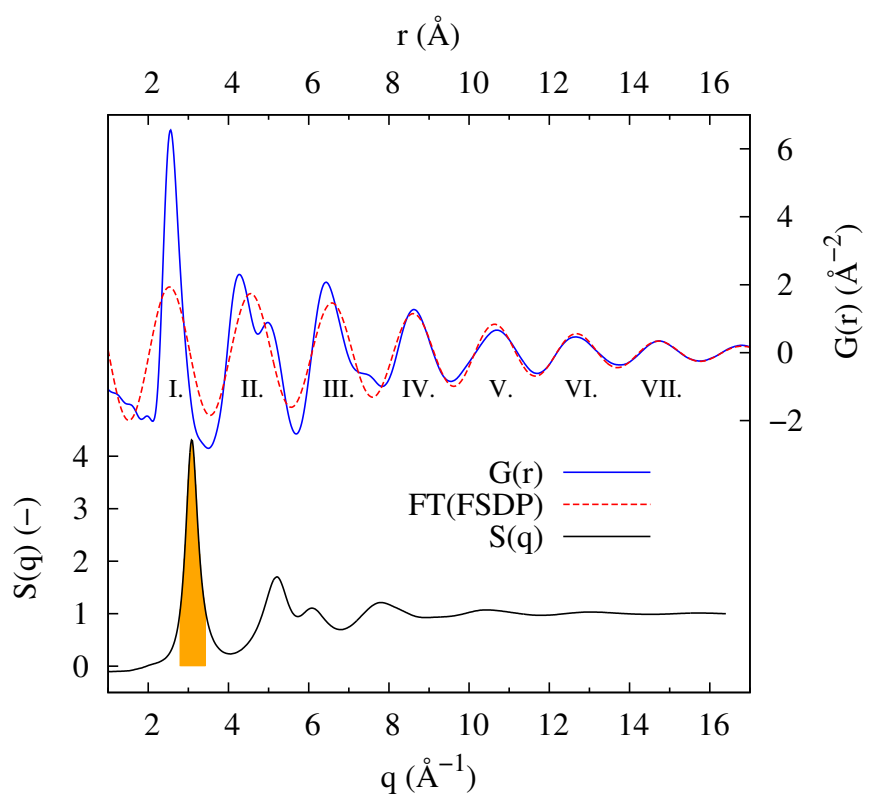

Fig. 6 (Color online) Structure factor $S(q)$ and reduced pair distribution function $G(r)$ for the $\mathrm{Fe}_{72.5} \mathrm{Cu}_{1} \mathrm{Nb}_{2} \mathrm{Mo}_{2} \mathrm{Si}_{15.5} \mathrm{~B}_{7}$ alloy in the as-quenched state. The red dashed line represents the result of the sine Fourier transformation of the FSDP appearing in $S(q)$ and covering a $q$-range $\langle 2.78,3.44\rangle \AA^{-1}$. Roman numerals denote respective coordination shells.

a function of temperature. The center of mass for the respective coordination shell (denoted by roman numerals in Fig. 6) was calculated using numerical integration. As can be seen from Fig. 7, the extent of thermal expansion strongly depends on the radial distance $r$, i.e. different shells exhibit different expansion rates ${ }^{31}$ (see Table 1). Similar results were observed in case of temperature dependence of the short-range order of $\mathrm{Cu}_{65} \mathrm{Zr}_{35}$ metallic glass ${ }^{32}$. Previous study on various metallic glasses undergoing tensile deformation has also shown that the atomic strain calculated from different coordination shells shows dependence on the radial distance $r^{33}$. Nevertheless all expansion curves presented in Fig. 7 show a change of the slope in the vicinity of the Curie temperature $T_{C}$. The observed $r$-dependence of the thermal expansion behavior is strongly correlated with the magnetic exchange interaction.

The ferromagnetism in amorphous materials is due to exchange interactions between the spins of neighboring unpaired electrons ${ }^{34}$. The interactions are stronger when the magnetic atoms are at short distance, while their separation, for example by metalloid atoms, makes the interactions weaker and, as a consequence, the magnetic saturation decreases. The exchange stiffness constant, $A$, can be obtained from the mean field model and from the Curie temperature ${ }^{35-37}$. When assuming Hasegawas model ${ }^{35}$ one can derive the following re- 

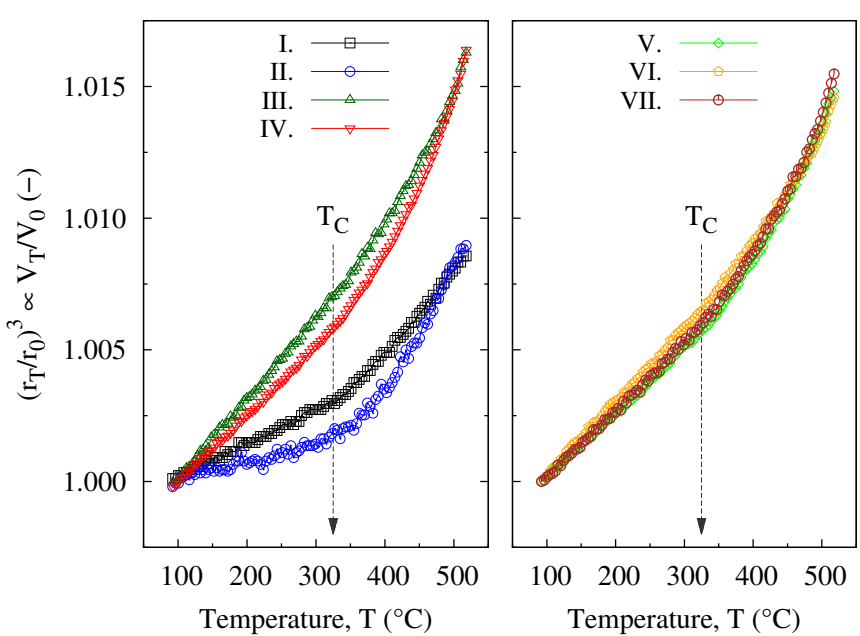

Fig. 7 (Color online) Relative change of the center of mass of the coordination shells I.-VII. during constant rate heating $(20 \mathrm{~K} / \mathrm{min})$ up to $520^{\circ} \mathrm{C}$. Corresponding coordination shells are depicted in Fig.6. Vertical arrows indicate the Curie point $T_{C}$.

Table 1 Comparison of coefficients of volume thermal expansion in ferromagnetic $\left(\alpha_{\text {ferro }}\right)$ and paramagnetic $\left(\alpha_{\text {para }}\right)$ state calculated by means of reciprocal and real space methods. The values of $\alpha_{\text {ferro }}$ and $\alpha_{\text {para }}$ were determined from linear fits to expansion curves on temperature intervals $\langle 100,320\rangle^{\circ} \mathrm{C}$ and $\langle 340,500\rangle^{\circ} \mathrm{C}$, respectively.

\begin{tabular}{llll}
\hline label & $\alpha_{\text {ferro }}\left[10^{-5} K^{-1}\right]$ & $\alpha_{\text {para }}\left[10^{-5} K^{-1}\right]$ & $\alpha_{\text {para }} / \alpha_{\text {ferro }}$ \\
\hline I. & $1.27 \pm 0.02$ & $2.98 \pm 0.04$ & $2.35 \pm 0.05$ \\
II. & $0.66 \pm 0.03$ & $4.03 \pm 0.10$ & $6.11 \pm 0.32$ \\
III. & $3.05 \pm 0.02$ & $4.47 \pm 0.05$ & $1.47 \pm 0.02$ \\
IV. & $2.57 \pm 0.01$ & $5.10 \pm 0.10$ & $1.98 \pm 0.04$ \\
V. & $2.43 \pm 0.01$ & $4.54 \pm 0.06$ & $1.87 \pm 0.03$ \\
VI. & $2.76 \pm 0.01$ & $3.84 \pm 0.03$ & $1.39 \pm 0.01$ \\
VII. & $2.56 \pm 0.01$ & $4.55 \pm 0.06$ & $1.78 \pm 0.02$ \\
FSDP & $2.41 \pm 0.05$ & $3.46 \pm 0.05$ & $1.44 \pm 0.04$ \\
\hline
\end{tabular}

lation for the exchange stiffness constant

$$
A=\frac{c_{F e} S_{F e} k_{B} T_{C}}{4\left(S_{F e}+1\right) r_{F e-F e}}
$$

where $c_{F e}$ is the concentration of $\mathrm{Fe}$ in atomic percent, $S_{F e}$ is the spin moment of $\mathrm{Fe}, T_{C}$ is the Curie temperature, $k_{B}$ is the Boltzmann constant and $r_{F e-F e}$ is the nearest-neighbor distance for $\mathrm{Fe}-\mathrm{Fe}$ atomic pairs. One can readily see from the equation that the exchange stiffness constant $A$ is inversely proportional to the interatomic distance $r$. From the relation $M=2 c_{F e} S_{F e} \mu_{B}$ in which $M$ is the magnetic moment expressed in Bohr magnetons $\mu_{B}$, one can calculate $S_{F e}$. When we consider the following values of the nearest-neighbor distance for $\mathrm{Fe}-\mathrm{Fe}$ atomic pairs, room temperature specific magnetization, density and Curie temperature $2.64 \AA, 130 \mathrm{Am}^{2} / \mathrm{kg}, 7.7 \mathrm{~g} / \mathrm{cm}^{3}$ and $325^{\circ} \mathrm{C}$, respectively, we obtain the value of the exchange

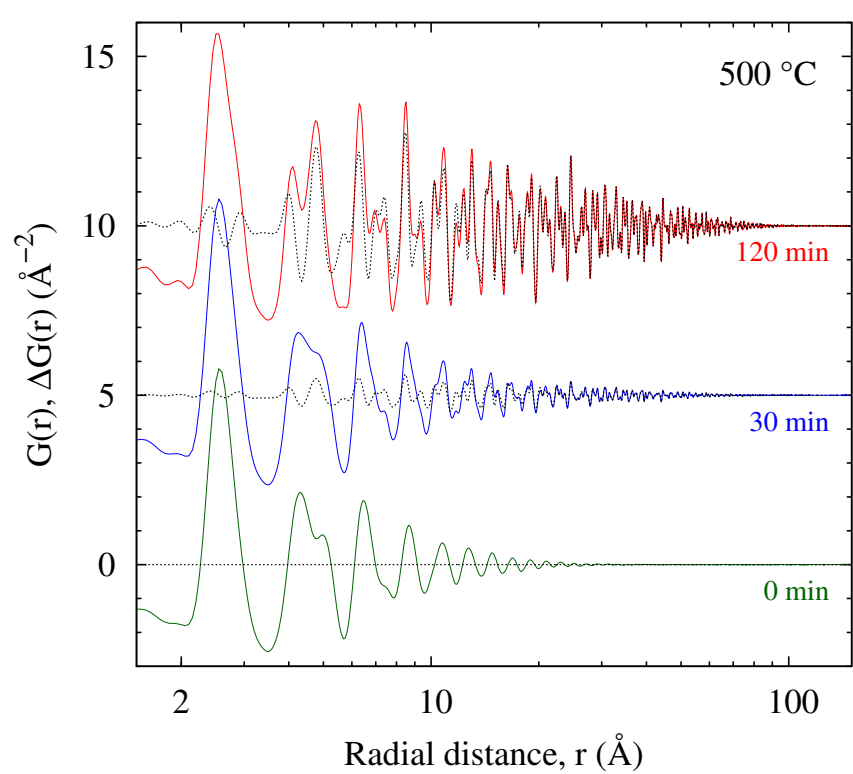

Fig. 8 (Color online) Comparison of pair distribution functions $G(r)$ at different times of isothermal annealing performed at $500^{\circ} \mathrm{C}$. Dotted lines correspond to $\Delta G(r)$ calculated according equation (19).

stiffness constant $A=(2.9 \pm 0.2) \times 10^{-12} \mathrm{~J} / \mathrm{m}$.

4.2.2 Crystallization kinetics The following section describes the kinetics of crystallization in real space by means of the pair distribution function $G(r)$. Figure 8 shows selected PDFs at various times of isothermal annealing at $500{ }^{\circ} \mathrm{C}$. With increasing annealing time $t$ oscillations on $G(r)$ become stronger and sharper. Also the distance $\delta$ at which oscillations fade out increases with annealing time $t$. All profile changes observed on PDFs are closely related to the formation of crystalline $\mathrm{Fe}_{3} \mathrm{Si}$ phase. To be more sensitive to temporal changes of the PDF during isothermal annealing we calculated the difference PDF, $\Delta G(r)_{t}$, in the following manner

$$
\Delta G(r)_{t}=G(r)_{t}-G(r)_{t=0}
$$

where $G(r)_{t}$ and $G(r)_{t=0}$ are corresponding to the PDF at a given time $t$ and reference time $t=0$, respectively. Kramer ${ }^{38}$ recently proposed an efficient way for the calculation of the difference PDF directly from difference of diffracted intensities $\Delta I(q)$. As can be seen from Fig. $8, \Delta G(r)_{t}$ reveals rather subtle changes in the nearest-neighborhood whereas medium and long-range order correlations show pronounced changes. It is worth noting that the difference PDF, $\Delta G(r)$, is almost identical with the total PDF, $G(r)$, for $r \geq 20 \AA$. Therefore in our further analysis we did the quantitative analysis of $\Delta G(r)_{t}$, which were calculated up to $r_{\max }=150 \AA$. Sharp oscillations appearing on the difference PDF are due to interatomic correlations within $\mathrm{Fe}_{3} \mathrm{Si}$ grains. Since grains are 


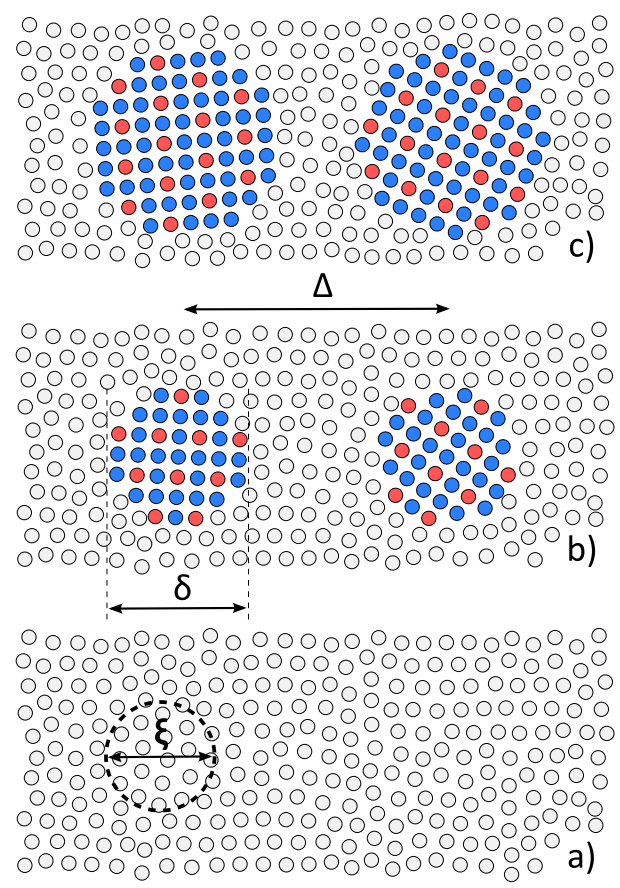

Fig. 9 (Color online) Schematic sketch showing the two-dimensional atomic structure obtained by isothermal annealing at $500{ }^{\circ} \mathrm{C}$ for a) $0 \mathrm{~min}$, b) $30 \mathrm{~min}$ and c) $120 \mathrm{~min}$. The gray dots depict atoms within the amorphous phase while the blue and the red dots represent $\mathrm{Fe}$ and $\mathrm{Si}$ atoms within the crystalline $\mathrm{Fe}_{3} \mathrm{Si}$ phase, respectively. The parameters $\xi, \delta$ and $\Delta$ represent correlation lengths characteristic for the amorphous phase, the interior of the individual $\mathrm{Fe}_{3} \mathrm{Si}$ grain and among two neighboring $\mathrm{Fe}_{3} \mathrm{Si}$ grains, respectively.

randomly distributed within the residual amorphous matrix, inter-grain atomic correlations effectively cancel out and thus the distance $\delta$ at which correlations fade out actually corresponds to the mean grain size $D^{39}$. The situation can be illustratively described by a simplified two-dimensional atomic structure shown in Fig. 9. At the beginning of the isothermal annealing the sample shows completely amorphous structure (Fig. 8, $t=0 \mathrm{~min}$ ). Due to lack of long-range order within the amorphous matrix, the maximum correlation length $\xi$ (i.e. the maximum distance up to which oscillations on PDF are detectable) is in the range between 20 and $30 \AA$ (Fig. 9a). After a specific incubation time (dependent on the temperature) the crystallization sets in and crystalline $\mathrm{Fe}_{3} \mathrm{Si}$ grains are formed. The early stages of crystallization are governed by the process of homogenous nucleation. The structural changes associated with the nucleation occur at length scales shorter than $\xi$ and therefore can be observed by the PDF in the nearest neighborhood ( $r$-range $\langle 2,5 \AA\rangle$ ) and medium range order ( $r$-range $\langle 5,20 \AA\rangle)$. As the grains increase their size the interatomic correlation length $\delta$ within $\mathrm{Fe}_{3} \mathrm{Si}$ grains gets larger than $\xi$

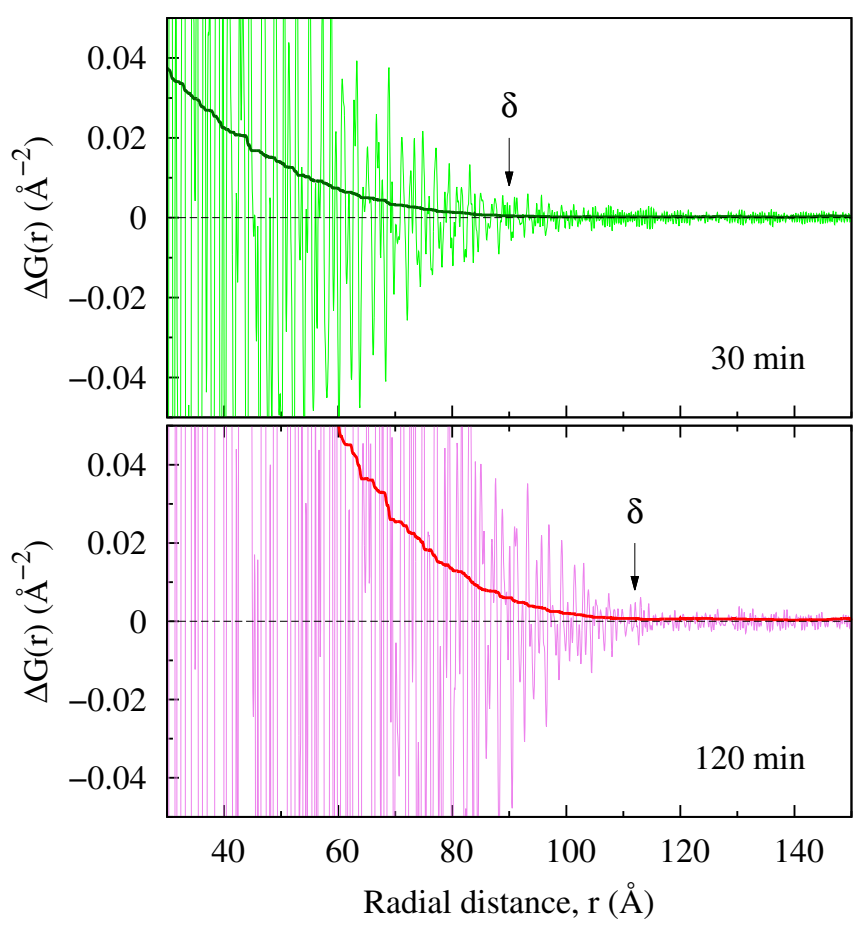

Fig. 10 (Color online) Detailed view on difference pair distribution functions $\Delta G(r)$ corresponding to annealing times 30 and $120 \mathrm{~min}$ at $500{ }^{\circ} \mathrm{C}$. Arrows indicate radial distance $\delta$ at which oscillations fade out. Solid lines correspond to respective cumulative distributions functions $C F(x)_{t}$ calculated according equation (20).

(Fig. 9b). Since $\mathrm{Fe}_{3} \mathrm{Si}$ grains are randomly distributed within the amorphous matrix the interatomic correlations among two neighboring $\mathrm{Fe}_{3} \mathrm{Si}$ grains over the distance $\Delta(\gg \delta)$ effectively cancel out due to broken inter-granular structural coherence. As a result the extent of oscillations in the PDF is related to the correlation length $\delta$ within $\mathrm{Fe}_{3} \mathrm{Si}$ grains which can be used to determine the mean grain size $D$. Further grain growth and typical microstructure coarsening is the dominant process during the late stages of crystallization (Fig. 9c).

One should keep in mind that the IRF in $q$-space determines the resolution of the PDF, namely in the high- $r$ region ${ }^{40}$. The experimental PDF is given as the product of the Fourier transform of the IRF and the ideal PDF obtained with infinite instrument resolution. The simplest approximation is that the IRF is a single Gaussian of constant width $\sigma_{q}$. The result from this model is a multiplication of the PDF by a broad Gaussian envelope function in real space with a width of $1 / \sigma_{q}{ }^{41}$. In our study diffraction data have only moderate $q$-space resolution $\left(\sigma_{q}=0.06 \AA^{-1}\right)$. This results in a loss of structural information in the high- $r$ region, for example beyond $r=120 \AA$ in the $\mathrm{LaB}_{6}$ PDF. In other words, the present experimental settings do not allow determination of grain sizes larger than $120 \AA$ from the PDF. Figure 10 shows a detailed view of the differ- 

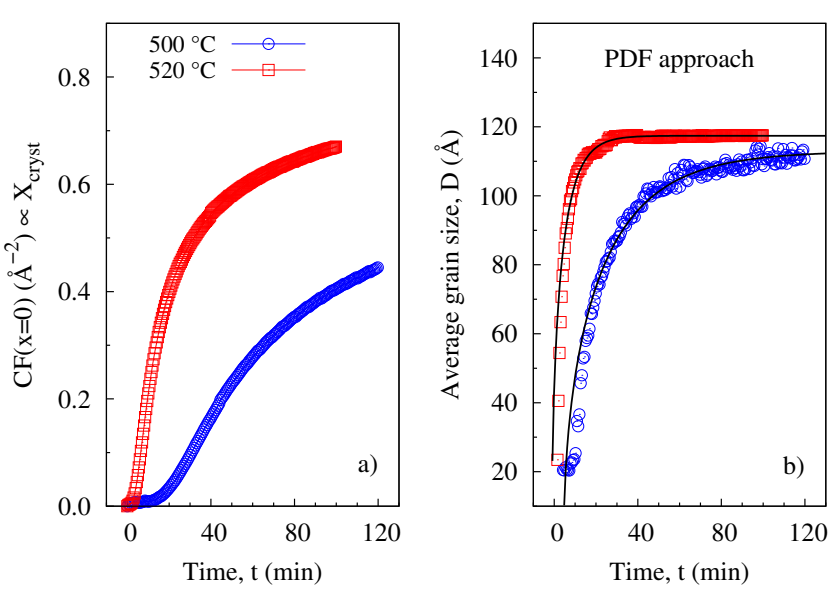

Fig. 11 (Color online) Temporal evolution of a) crystalline fraction and b) mean grain size as inferred form the difference PDFs by means of the cumulative function $C F(x)$. Solid lines represent fits to equation (21). The uncertainty in determination of the mean grain size is $\pm 5 \AA$.

ence pair distribution functions $\Delta G(r)$ corresponding to annealing times 30 and $120 \mathrm{~min}$ at $500^{\circ} \mathrm{C}$. In order to determine the distance $\delta$ at which oscillations on $\Delta G(r)$ fade out we propose the following cumulative function

$$
C F(x)_{t}=\sqrt{\frac{1}{\left(r_{\max }-x\right)} \int_{x}^{r_{\max }}\left[\Delta G(r)_{t}\right]^{2} d r}
$$

where $x$ is any value from $\langle 0,150 \AA\rangle, t$ is the time and $r_{\max }=$ $150 \AA$. The $C F(x)$ function defined by the above equation actually calculates root mean square (RMS) value of the $\Delta G(r)$ on the interval $\langle x, 150\rangle$. It is worth noting that the $C F(x)$ is monotonically decreasing and above $\delta$ significantly drops in value and flattens due to missing structural oscillations in the high- $r$ region. To determine the mean grain size $D$ from $\Delta G(r)$ one can simply find the value of radial distance at which $C F(x)$ drops below the noise level calculated from the high- $r$ region. Since the fraction of crystalline phase is proportional to the amplitudes of oscillations on $\Delta G(r)$ we suggest it can be directly calculated from $C F(x)$ by putting $x=0$. Figure 11 shows the temporal evolution of the crystalline fraction and mean grain size as inferred from the difference PDFs by means of the cumulative function $C F(x)$. The fractions $X_{\text {cryst }}$ of crystalline $\mathrm{Fe}_{3} \mathrm{Si}$ phase calculated from reciprocal space (Fig.4a) and real space (Fig.11a) are showing qualitatively almost identical time dependence. When comparing the average grains size $D$ determined from PDF in real space (Fig.11b) and from XRD profiles in reciprocal space (Fig.5) one can readily see that the PDF method offers much more resolving power for determining grain sizes well below $80 \AA$.
Following the work of Zuo et al. ${ }^{42}$ the kinetics of the grain growth can be expressed in the form

$$
\frac{D^{2}(t)-D_{\max }^{2}}{D^{2}(0)-D_{\max }^{2}}=\exp \left[-\frac{2 k t}{D_{\max }^{2}}\right]
$$

where $D(t), D(0)$ and $D_{\max }$ is the average grain size at any time $t, t=0$ and $t \rightarrow \infty$, respectively, the rate constant $k$ is expressed in the Arrhenius form

$$
k=k_{0} \exp \{-Q / R T\}
$$

where $R$ is the gas constant, $T$ is absolute temperature, $k_{0}$ is a constant and $Q$ the activation energy for grain growth. Fitting the data presented in Fig. $11 \mathrm{~b}$ to equation (21) we obtained the rate constant values $228 \pm 8$ and $928 \pm 13 \AA^{2} \mathrm{~min}^{-1}$ for isothermal annealing at 500 and $520^{\circ} \mathrm{C}$, respectively. The activation energy for grain growth, $Q$, calculated using equation (22) is $357 \pm 12 \mathrm{~kJ} / \mathrm{mol}(3.70 \pm 0.12 \mathrm{eV} /$ atom).

\section{Conclusions}

Constant heating rate annealing provided information about the thermal expansion of an amorphous phase. We found that the change of the slope of the thermal expansion curve is directly related to the ferromagnetic-to-paramagnetic phase transition. Real space analysis by means of PDF revealed that the rate and extent of thermal expansion depend on the interatomic separation. The temporal behavior of microstructural parameters such as lattice parameter, mean grain size and relative fraction of crystalline phase provides insights into the kinetics of nanocrystallization during isothermal holding. Both approaches, reciprocal and real space methods, reveal similar qualitative results. Analyzing XRD profiles in reciprocal space is straight forward and relatively easy to perform. However, assuming a proper model for line broadening is essential in getting reliable estimate of the mean grain size. Also the relative fraction of crystalline phase is strongly affected by choosing an appropriate $2 \theta$ range. On the other hand, calculations of the structure factor and pair distribution function are more computationally demanding but there are no further assumptions needed for determination of the mean grain size. We propose that the root mean square of the difference PDF can be successfully used to extract information about the mean grain size and fraction of crystalline phase. When comparing with the Williams-Hall method in reciprocal space, the PDF method offers much more resolving power to determine size of ultra-fine particles which are having grain sizes well below $80 \AA ̊$. Following grain growth kinetics during isothermal annealing at 500 and $520^{\circ} \mathrm{C}$ we found that the activation energy of the process is $357 \pm 12 \mathrm{~kJ} / \mathrm{mol}$. 


\section{Acknowledgements}

The authors wish to express their thanks to O. Gutowski for great technical assistance during beamtime at P07 beamline. The samples were kindly provided by S. Roth from IFW Dresden. This work was partially supported by the Slovak Grant Agency for Science Nr. 1/0148/12 and by the DESY proposal Nr. I20110689 EC. J.B. acknowledges financial support from Zhejiang University-Helmholtz cooperation fund. S.M. acknowledges financial support from DAAD agency. V.K. acknowledges financial support through the National Scholarship Programme of the Slovak Republic.

\section{References}

1 Y. Yoshizawa, S. Oguma and K. Yamauchi, J. Appl. Phys., 1988, 64, 6044.

2 M. E. McHenry, M. A. Willard and D. E. Laughlin, Prog Mater Sci, 1999, 44, 291.

3 Y. Yoshizawa and K. Yamauchi, IEEE Trans. Magn., 1989, 25, 3324.

4 J. Petzold, J. Magn. Magn. Mater, 2002, 242-245, 84.

5 G. Herzer, IEEE Trans. Magn., 1989, 25, 3327.

6 G. Herzer, in NATO Science Series II: Mathematics, Physics and Chemistry, ed. B. Idzikowski, P. Svec and M. Miglierini, Kluwer Academic, Dordrecht, 2005, vol. 184, ch. The Random Anisotropy Model - A critical Review and Update, p. 15

7 C. Conde, M. Millan, J. Borrego, A. Conde, M. Capitan and J. L. Joulaud, Philosophical Magazine Letters, 1998, 78, 221.

8 E. Illekova, Thermochimica Acta, 2002, 387, 47.

9 P. J. Chupas, X. Qiu, J. C. Hanson, P. L. Lee, C. P. Greya and S. J. L. Billinge, J. Appl. Cryst., 2003, 36, 1342.

10 S. J. L. Billinge and M. G. Kanatzidis, Chem. Commun., 2004, 749.

11 D. Holland-Moritz, F. Yang, T. Kordel, S. Klein, F. Kargl, J. Gegner, T. Hansen, J. Bednarcik, I. Kaban, O. Shuleshova, N. Mattern and A. Meyer, EPL (Europhysics Letters), 2012, 100, 56002.

12 J. Bednarcik, S. Michalik, M. Sikorski, C. Curfs, X. D. Wang, J. Z. Jiang and H. Franz, Journal of Physics: Condensed Matter, 2011, 23, 254204.

13 A. Hammersley, S. Svensson, M. Hanfland, A. Fitch and D. Häusermann, High Press. Res., 1996, 14, 235.

14 P. Scherrer, Gottinger Nachrichten Gesell., 1918, 2, 98.

15 G. Williamson and W. Hall, Acta Metall., 1953, 1, 22.

16 C. Suryanarayana and M. G. Norton, X-ray Diffraction: A Practical Approach, Plenumm Press, New York, 1998.

17 F. R. G. Caglioti, A. Paoletti, Nucl. Instrum. Methods, 1958, 3, 223.

18 P. Riello, P. Canton and G. Fagerazzi, J. Appl. Cryst., 1998, 31, 78.

19 H. Balyuzi, Acta Cryst. A, 1975, 31, 600.

20 D. Wassmaier and A. Kirfel, Acta Cryst. A, 1995, 51, 413.

21 T. Egami and S. Billinge, Underneath the Bragg Peaks: Structural analysis of complex materials, Pergamon Press, Elsevier, Oxford, England, 2003.

22 Y. Waseda, The Structure of Non-Crystalline Materials, McGraw-Hill Inc., 1980.

23 B. Thijsse, J. Appl. Cryst., 1984, 17, 61-76.

24 T. Faber and J. Zimman, Philos. Mag., 1965, 11, 153.

25 A. Yavari, A. Moulec, A. Inoue, N. Nishiyama, N. Lupu, E. Matsubara, W. Botta, G. Vaughan, M. Michiel and A. Kvick., Acta Mater., 2005, 53, 1611.

26 S. Michalik, J. Bednarcik, J. Kovac, P. Sovak and H. Franz, J. Phys. D: Appl. Phys., 2012, 45, 455302 (5pp).
27 S. Michalik, J. Gamcova, J. Bednarcik and R. Varga, Journal of Alloys and Compounds, 2011, 509, 3409.

28 W. Li, H. Li, H. Sun, J. Zhang and X. Zhang, Appl. Phys. Lett., 2005, 86, 092501.

29 J. Blazquez, S. Roth, C. Mickel and A. Conde, Acta Mater., 2005, 53, 1241.

30 S. Polizzi, G. Fagherazzi, A. Benedetti, M. Battagliarin and T. Asano, J. Appl. Cryst., 1990, 23, 359.

31 Extra Supplementary Information (ESI), For more deatils see ESI.

32 N. Mattern, J. Bednarcik, M. Stoica and J. Eckert, Intermetallics, 2013, 32, $51-56$.

33 X. D. Wang, J. Bednarcik, H. Franz, H. B. Lou, Z. H. He, Q. P. Cao and J. Z. Jiang, Applied Physics Letters, 2009, 94, 011911.

34 E. Kneller, Ferromagnetismus, Springer-Verlag Berlin, 1962.

35 R. Hasegawa, J. Appl. Phys., 1974, 45, 3109.

36 N. Heiman, K. Lee, R. I. Potter and S. Kirkpatrick, J. Appl. Phys., 1976, 47, 2634.

37 H. Lassri, L. Dirouch and R. Krishnan, J. Appl. Phys., 1994, 75, 6309.

38 M. Kramer, J. Appl. Cryst., 2007, 40, 77.

39 T. Proffen, S. Billinge, T. Egami and D. Louca, Z. Kristallogr., 2003, 218, 132.

40 B. H. Toby and T. Egami, Acta Cryst., 1992, A48, 336.

41 X. Qiu, E. S. Bozin, P. Juhas, T. Proffen and S. J. Billinge, J. Appl. Cryst., 2004, 34, 110.

42 B. Zuo and T. Sritharan, Acta Mater., 2005, 53, 1233. 